\title{
PAPERS
}

\section{Dementia due to tumours of the corpus callosum}

\author{
M. J. G. HARRISON \\ D.M., F.R.C.P. \\ Institute of Neurological Studies, Middlesex Hospital, Mortimer Street, London WI
}

\begin{abstract}
Summary
The clinical features of $\mathbf{1 6}$ cases of tumours of the corpus callosum are described. Many presented with mental changes which were eventually obvious in all but one of the patients. The need to consider these tumours in the differential diagnosis of dementia is discussed.
\end{abstract}

KEY WORDS: papilloedema, computed tomographic scan, epilepsy.

\section{Introduction}

Surveys of large numbers of patients presenting with dementia (e.g. Marsden and Harrison, 1972) reveal that progressive intellectual deterioration is due to a cerebral tumour in some $5-10 \%$. Frontal meningiomas, multiple metastases, and temporal gliomas predominate but a recently completed survey has emphasized how frequently tumours of the corpus callosum cause dementia.

\section{Patients and methods}

Over the course of a 5-year-period (1978-1983), 16 patients with corpus callosum tumours were encountered either at the National Hospital, Queen Square or at the Middlesex Hospital. In each case the diagnosis was made by computed tomographic (CT) scanning. It was confirmed by biopsy or at autopsy in 11 instances. A retrospective case note survey concentrated on the clinical presentation and mental state examination, together with a review of the results of psychometry where this had been carried out.

\section{Results}

There were 10 male and six female patients from 9-68 years though $70 \%$ were between 50 and 68 years at the time of presentation. Seven patients had a short illness of less than 1 month. Five gave a history of 1-3 months, and four had a longer duration of symptoms of up to 12 months. The initial complaint is shown in Table 1. Five patients presented with headache with or without vomiting. Three patients commenced with seizures and two with limb or gait disurbance. Six patients however presented with mental change. In three cases the complaint from patient or family was of increasing forgetfulness, in one instance of 12 months duration. In the others the families described apathy and lethargy and a rather depressed withdrawn state. Two patients had become irritable, 'fussy' and anxious. These early symptoms had clearly been thought to represent the effects of depression or of progressive degenerative conditions.

TABLE 1. Presenting and eventual symptoms in 16 patients with tumours of the corpus callosum

\begin{tabular}{lcc}
\hline Presenting complaints & & Eventual symptoms \\
\hline Mental change & 6 & 15 \\
Headache and vomiting & 5 & 12 \\
Epilepsy & 3 & 5 \\
Ataxic gait & 1 & 2 \\
Limb symptoms & 1 & 4 \\
Aural symptoms & - & 3 \\
Bladder symptoms & - & 2 \\
Diplopia & - & 1 \\
\hline
\end{tabular}

By the time of admission to hospital for investigation 15 out of the 16 patients had a history of mental change. Two were recorded to be simply confused and/or disorientated, two more as apathetic. One was disinhibited and 'giggly'. Two were drowsy as well as confused, and eight had a poor memory (Table 1). Headache had developed in the majority (10), epilepsy in five, and limb or gait disorder in six. One patient developed diplopia. Two had bladder problems, in one case incontinence, in the other urinary retention of which the patient was unaware or unconcerned. Three patients had aural complaints of pain and tinnitus.

The examination (Table 2 ) revealed bedside evi- 
dence of dementia (poor memory and learning, disorientation and poor general knowledge) in 12 patients. One was noted to be slow, and one showed disinhibition and emotional lability. One patient was too drowsy for further assessment. Only one patient was thought to have a normal mental state.

TABLE 2. Findings on clinical examination in 16 patients with tumours of the corpus callosum

\begin{tabular}{lr} 
Demented & 12 \\
Papilloedema & 9 \\
Bilateral extensor plantar responses & 7 \\
Hemianopia & 5 \\
Hemisensory inattention & 4 \\
Primitive reflexes & 4 \\
Hemiparesis & 3 \\
Neck stiffness & 2 \\
Drowsy & 2 \\
\hline
\end{tabular}

Evidence of raised intracranial pressure was found in nine patients with papilloedema. Two had neck stiffness probably due to tonsillar herniation (confirmed at autopsy in one). Six patients showed parietal signs (hemianopia and sensory hemi-inattention); and seven had bilateral extensor plantar responses. Three had a mild or moderate hemiparesis.

Psychometry had been carried out in eight cases. All had memory impairment and either a performance/verbal discrepancy or subtest evidence of deterioration. Four patients had additional evidence of dysphasia or dyslexia. Two patients had bilater $\overrightarrow{9}$ selective deficits. Specific testing for evidence of disconnection was rarely carried out but one patien was noted to read faster in the right half of the visuas field than the left.

CT scans in all cases showed a mass in the corpust callosum, commonly a butterfly tumour (Fig. 1). One patient's scan showed a lipoma with partial agenesis of the corpus callosum. Biopsies in nine patient confirmed the presence of a glioma, found in tw additional cases at autopsy.

\section{Discussion}

In 1884 Bristowe described four patients with tumours of the corpus callosum. The point of hise article was to stress the peculiar mental state of his patients who also had a progressive hemiparesis. The first detailed discussion of the psychological symp. toms is usually however attributed to Raymond is 1906. At about the time of introduction of ventricul ography Ironside and Guttmacher (1929) and Voris and Adson (1935) both described larger series of patients. It is clear from their case material that mental changes were virtually always present, an $\$$ commonly early in appearance. Poor memory and apathy predominated and were more common tha in tumours elsewhere in the hemispheres.

The diagnosis was rarely made in life during the period covered by these classical descriptions hơp ever. Earlier detection is now possible particularrly since the advent of computerized axial tomograpipe The present study reveals that apathy, mental düls

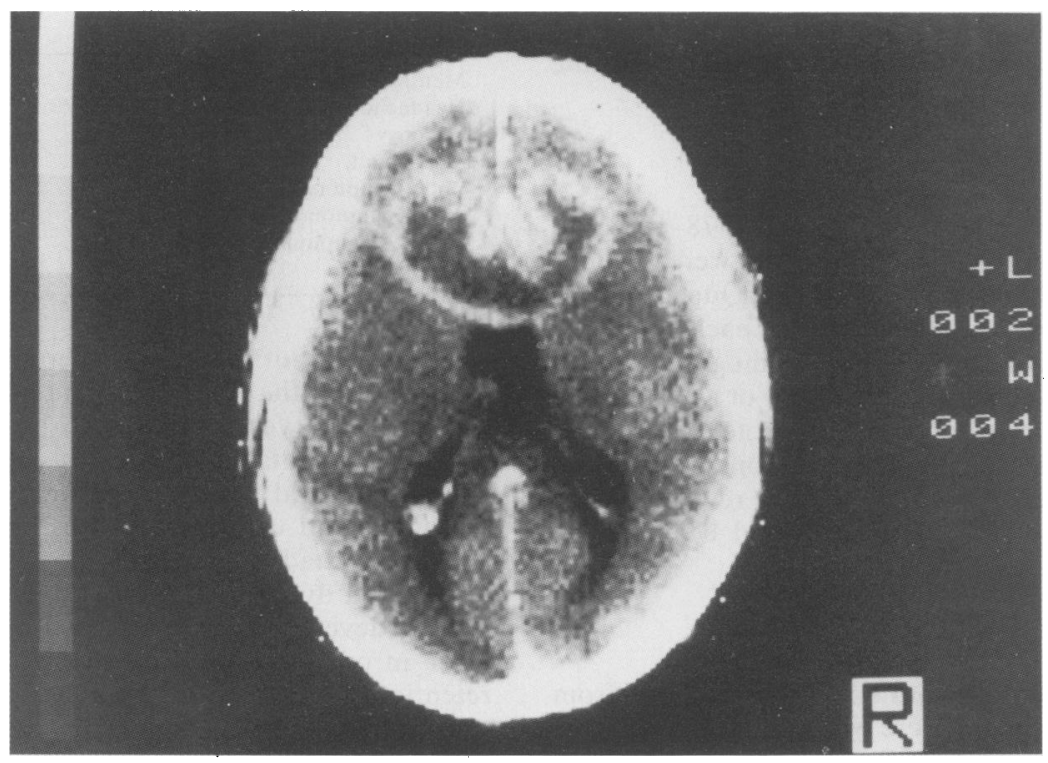

FIG. 1. CT scan showing a butterfly glioma of the corpus callosum. 
ness and poor short-term memory remain common presenting symptoms in cases of callosal tumours diagnosed by CT scanning. Psychometry confirms that the changes are usually of diffuse deterioration of personality, intellect and memory. By the time of the study, subtle signs of hemisphere disconnection are probably undetectable because of the severity of the progressive dementia. The picture is virtually indistinguishable from that of Alzheimer's disease in many instances.

By the time of admission to hospital most of this series of patients had developed further features enabling a diagnosis of cerebral tumour to be made. It is suggested that a corpus callosum tumour be considered in any middle-aged patient with a short history of dementia. Any evidence of associated headache or early focal signs increases the chances of such a diagnosis.

\section{Acknowledgment}

I am grateful to Professor John Marshall and to the physicians and surgeons of the Middlesex Hospital and the National Hospital Queen Square for permission to study patients admitted under their care.

\section{References}

BRISTOWE, J.S. (1884) Tumour of the corpus callosum. Brain, 7, 315. IRONSIDE, R. \& GUTTMACHER, M. (1929) The corpus callosum and its tumours. Brain, 52, 442.

MARSDEN, C.D. \& HARRISON, M.J.G. (1972) The outcome of investigation of presenile dementia. British Medical Journal, 2, 249.

RAYMOND, F. (1906) Quoted by Ironside and Guttmacher (1929).

VORIS, H.C. \& ADSON, A.W. (1935) Tumours of the corpus callosum. Archives of Neurology and Psychiatry, 34, 965.

(Accepted 6 September 1983) 